\title{
Management of Simultaneous Symptomatic Bilateral Carotid Stenosis
}

\author{
Jessica Lynn Zambonin, Gwynedd E. Pickett
}

Keywords: Carotid artery disease, TIA, Carotid endarterectomy, Endovascular, Stroke Prevention, Vascular neurology

A 49-year-old right-handed woman with a history of hypertension, dyslipidemia, and myocardial infarct presented to the emergency department with symptoms of bilateral transient ischemic attack. She was experiencing right hemifacial numbness of sudden onset. However, she reported having had an episode of left arm weakness and numbness earlier that day, associated with difficulty recalling numbers. She continued to describe some numbness in her left hand.

Her medication included enteric-coated ASA (81 mg daily), telmisartan/hydrochlorothiazide $(80 / 12.5 \mathrm{mg}$ daily), extended release diltiazem (120 mg daily), and fluticasone/salmeterol (250/ $50 \mathrm{mcg}$ inhalation twice daily). She did not drink alcohol and had quit smoking 6 months before, with a previous 34-pack-year history. She did not report any prior stroke or transient ischemic attack.

Examination revealed an overweight woman in no distress. Her blood pressure was $145 / 75 \mathrm{mmHg}$ and heart rate 88 beats per minute and regular. Her cardiac examination was normal. Neurological examination was notable only for reportedly impaired sensation over the right face and scalp as well as the left hand and forearm, both of which subsequently resolved.

A computed tomography scan of the brain revealed old and new ischemic changes in both cerebral hemispheres. Carotid Doppler ultrasound suggested severe bilateral carotid stenosis involving both the right and left proximal internal carotid arteries, with possible near occlusion. Computed tomography angiography revealed $81 \%$ stenosis of the right internal carotid and $76 \%$ stenosis of the left internal carotid by North American Symptomatic Carotid Endarterectomy Trial (NASCET) criteria. The left posterior communicating artery was not seen; the right posterior communicating artery and anterior communicating artery were present. Diffusion-weighted magnetic resonance imaging revealed bilateral early subacute white matter infarcts with evidence of prior small infarcts. Electroencephalograph showed normal sinus rhythm.

She underwent left carotid endarterectomy within two weeks of presentation, without complications. Seven days later, she underwent right carotid endarterectomy, again without complications. Both procedures were performed under general anaesthesia, employing two-channel electroencephalograph monitoring and measurement of stump pressures. She made a good recovery and had no further neurological events. Aggressive risk factor modification was undertaken.

\section{DISCUSSION}

Stroke is a leading cause of morbidity and mortality in Canada, with carotid stenosis accounting for $10 \%$ to $15 \%$ of all ischemic strokes. ${ }^{1,2}$ Current management of carotid stenosis includes optimizing medical therapy, with or without carotid revascularization, to lower the risk of future stroke. Best medical therapy is the least invasive treatment option and typically involves antiplatelet medication combined with reducing modifiable vascular risk factors including hypertension and dyslipidemia. The gold standard for revascularization is carotid endarterectomy (CEA) a surgical procedure that involves excision of the atherosclerotic lesion. Recently, carotid angioplasty and stenting (CAS) has emerged as a less invasive endovascular alternative for revascularization. Choice of therapy depends on the presence or absence of stroke-like symptoms (symptomatic vs asymptomatic), the degree of stenosis (mild, moderate, severe), and the risks associated with intervention. ${ }^{1}$

Here we present a case in which a patient presented with severe bilateral carotid stenosis with the unique attribute of both stenoses causing recent symptoms and requiring timely intervention. Simultaneous symptomatic carotid disease is a diagnosis of exclusion. Bilateral cerebral ischemic events should prompt investigation of a cardiac source of emboli. Additionally, anomalies of the circle of Willis may also produce bilateral hemispheric events from emboli arising from a single carotid plaque. There are no management guidelines for patients with simultaneous symptomatic bilateral carotid stenosis. It is common for patients presenting with unilateral symptomatic carotid artery disease to have varying degrees of stenosis in the contralateral artery; however, it is rare for both sides to cause symptoms simultaneously. Bilateral symptoms more frequently may result from a cardiac source, unilateral carotid stenosis in the setting of an incomplete circle of Willis, or a brainstem event.

Based on current Canadian Stroke Best Practice Recommendations, patients with severe internal carotid stenosis should be offered CEA as soon as possible with the goal of operating within 14 days. A surgeon with a known perioperative morbidity and mortality rate of less than $6 \%$ should be selected to perform the procedure. $^{2}$ These recommendations are based on best evidence from large multicenter randomized control trials, particularly NASCET and European Carotid Surgery Trial, which evaluated

\footnotetext{
From the Division of Neurosurgery, Dalhousie University, Halifax, Nova Scotia, Canada. Received May 21, 2014. Final Revisions Submitted February 20, 2015. Correspondence to: Jessica Lynn Zambonin, Dalhousie University - Division of Neurosurgery, 1796 Summer Street, Halifax, Nova Scotia B3H 3A7, Canada. Email: jessica.zambonin@gmail.com
} 
the benefit of medical and surgical intervention in reducing the risk of future stroke. ${ }^{3}$

A subgroup analysis in NASCET evaluated the effects of contralateral stenosis on the benefit of CEA. Patients with symptomatic ipsilateral severe stenosis were shown to benefit from surgery on the symptomatic artery even in the presence of contralateral severe stenosis, with a two-year reduced risk of stroke of more than $50 \%$ compared with medical therapy alone. ${ }^{4}$ These patients were more likely to experience perioperative stroke or death in surgery group compared with those without contralateral stenosis, but the benefits in terms of stroke prevention still outweighed the increased risk. ${ }^{4}$ This trial did not report any cases in which the patient had bilaterally symptomatic carotid arteries.

CAS has appeal as a minimally invasive procedure without some of the risks associated with CEA, such as cranial nerve injury or surgical site hematoma. Multiple trials have compared stenting to surgery (Sorafenib or placebo in combination with transarterial chemoembolization, Endarterectomy versus Angioplasty in Patients with Symptomatic Severe Carotid Stenosis, Carotid and Vertebral Artery Transluminal Angioplasty Study, Stenting and Angioplasty with Protection in Patients at High Risk for Endarterectomy, Carotid Revascularization Endarterectomy vs. Stenting Trial), with varying results. None has shown a clear advantage to CAS, although Carotid Revascularization Endarterectomy vs. Stenting Trial suggested that for patients $<65$ years of age CAS had a lower complication rate than CEA. 5 A metaanalysis of 16 trials was published in 2012 that contrasted endovascular treatment and surgery. Results indicated no significant difference in the reduction of future stroke in any territory. ${ }^{1}$ However, of the studies included, only one trial reported event rates beyond five years. Long-term follow-up reports from the Carotid and Vertebral Artery Transluminal Angioplasty Study investigators found that restenosis, with associated clinical symptoms, was three times higher in the endovascular group. ${ }^{1}$ The risk of restenosis is particularly relevant to younger patients. The Canadian guidelines currently advise that carotid stenting may be considered for patients who are not operative candidates for technical, anatomic, or medical reasons. ${ }^{2}$ The guidelines do not list specific contraindications, but many articles cite the criteria proposed by the Stenting and Angioplasty with Protection in Patients at High Risk for Endarterectomy trial. These include clinically significant cardiac disease, severe pulmonary disease, contralateral carotid occlusion, contralateral laryngeal nerve palsy, recurrent stenosis after CEA, previous radical neck surgery or radiation therapy to the neck, and an age greater than 80 years. ${ }^{1}$ Our patient was not felt to meet the high-risk criteria; she did have a history of myocardial infarct but did not currently have clinically significant disease. Therefore, based on current guidelines and best evidence, CEA was recommended to our patient.

Most reports of bilateral CEA suggest a staged rather than a simultaneous approach. ${ }^{6}$ Proponents of the staged method suggest there may be increased neurological complications associated with operating on both carotids simultaneously. Potential bilateral damage to cranial nerves is another risk, particularly in the case of the hypoglossal or recurrent laryngeal nerves. Staging the surgeries allows functional assessment of these nerves before proceeding to the contralateral side. The likelihood of hyperperfusion syndrome, resulting from increased blood flow post-CEA, also poses a potential additive risk if both plaques are removed simultaneously. This condition has been shown to lead to paralysis or severe impairment of cerebral autoregulation, which may result in intracerebral haemorrhage and increased postoperative mortality. ${ }^{6}$ Although simultaneous CEA carries the advantages of avoiding repeated anesthesia and potentially diminishing the psychological stress on the patient, these benefits likely do not outweigh the potential risks associated with nerve injury and hyperperfusion syndrome. Recent small studies have reported that perioperative risks for staged bilateral CEA are typically lower than $5 \%{ }^{6}$ Therefore, based on a perioperative risk of $5 \%$, the staged approach for CEA would remain within the indications proposed by Canadian best practice recommendations for stroke care.

We elected to stage our patient's operations one week apart. This was considered long enough for any postoperative complications to manifest, in addition to allowing sufficient recovery before the subsequent operation, while not overly delaying treatment for one of the symptomatic arteries.

The final question regarding management strategy for our patient was which carotid artery would receive priority. Because the patient was right-handed, it was felt that a stroke involving her dominant left hemisphere would be the most potentially devastating. In discussions with her, it was agreed to treat the left side first.

This case report illustrates that bilateral carotid stenosis is a treatable condition in which staged carotid endarterectomy is a safe option for patients presenting with transient ischemic attack. Simultaneous symptomatic presentations of bilateral carotid stenosis is a rare occurrence, and decisions regarding management may be extrapolated from related clinical trials, strengthened with evidence from individual case reports and clinical judgment.

\section{Disclosures}

JLZ does not have anything to disclose. GP does not have anything to disclose.

\section{REFERENCES}

1. Bonati L, Lyrer P, Ederle J, et al. Percutaneous transluminal balloon angioplasty and stenting for carotid artery stenosis. Cochrane Database Syst Rev. 2012;9:CD000515.

2. Coutts SB, Wein TH, Lindsay MP, et al. Canadian Stroke Best Practice Recommendations: secondary prevention of stroke guidelines, update 2014. Int J Stroke. 2015;10:282-91.

3. Rerkasem K, Rothwell P. Carotid endarterectomy for symptomatic carotid stenosis. Cochrane Database Syst Rev. 2011;4:CD001081.

4. Gasecki A, Eliasziw M, Ferguson G, et al. Long-term prognosis and effect of endarterectomy in patients with symptomatic severe carotid stenosis and contralateral carotid stenosis or occlusion: results from NASCET. J Neurosurg. 1995;83:778-82.

5. Voeks JH, Howard G, Roubin GS, et al. Age and outcomes after carotid stenting and endarterectomy: the Carotid Revascularization Endarterectomy versus Stenting Trial. Stroke. 2011;42:3484-3490.

6. Dimakakos P, Kotsis T, Tsiligiris B, et al. Comparative results of staged and simultaneous bilateral carotid endarterectomy: a clinical study and surgical treatment. Cardiovasc Surg. 2000;8:10-7. 